\title{
A New Repetitive Control Scheme Based on Non-Causal FIR Filters
}

\author{
Yik R. Teo ${ }^{1}$ and Andrew J. Fleming ${ }^{1}$
}

\begin{abstract}
Repetitive Control (RC) is a popular technique for tracking periodic signals and rejecting periodic disturbances. Repetitive control achieves accurate tracking of periodic trajectories by incorporating a periodic signal generator within the feedback loop. The periodic signal generator provides an infinite loop-gain at the harmonic frequencies of the reference signal. However, this scheme cannot be used in isolation due to challenges with stability and robustness. The stability and robustness can be improved by incorporating appropriate filters. However, there is a trade-off between robustness and tracking performance. The current state-of-the art is to implement plant inversion and include phase compensators to improve the highfrequency tracking performance. In this work, a RC controller is combined with a non-causal FIR filter to improve the tracking performance without the requirement for phase compensators or plant inversion. The performance of the proposed $\mathrm{RC}$ design is demonstrated on a piezoelectric positioner.
\end{abstract}

\section{INTRODUCTION}

Repetitive control ( $\mathrm{RC})$ is a learning-type control technique which "learns" from experience to improve the control performance [1]. The technique has been widely used in various engineering application such as robotics [2], hard-disk arm actuators [3], electro-hydraulics [4], nanopositioning [5], [6] and optical disk drives [7]. The idea of repetitive control was originally developed to reject the periodic disturbances in a power supply control application [8] and to track periodic references in motion control applications [9].

Repetitive control is based on the Internal Model Principle (IMP) that was first formalized in [10]. This principle states that if a certain signal must be rejected or tracked with zero steady-state error, the generator must be inside the control loop, in the controller, or in the plant itself. Any periodic signal with a known period L can be generated by a controller which has positive feedback around a pure time delay, as illustrated in Fig. 1. The result is a controller with an infinite number of marginally-stable poles with infinite gain at the harmonics of the periodic reference. Compared to traditional feedback and feed-forward controllers, the tracking error of RC reduces as the number of operating periods increases. The repetitive control technique in discrete-time is shown in [11].

The major challenges of RC control are stability, robustness, and steady-state tracking performance. The problems with stability and robustness arise from the interaction between the high frequency RC gain with the plant dynamics. However, this problem can be avoided by incorporating appropriate filters in the RC loop [1], [12]-[17]. However, a

\footnotetext{
${ }^{1}$ Yik R. Teo and Andrew J. Fleming are with the Precision Mechatronics $\mathrm{Lab}$ at the School of Electrical Engineering and Computer Science, The University of Newcastle, Callaghan, New South Wales, 2308, Australia [yik.teo, andrew.fleming] @newcastle.edu.au
}

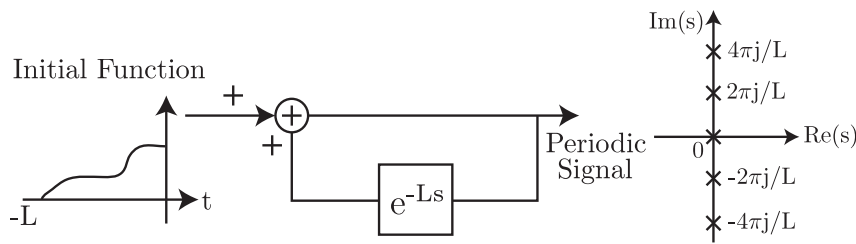

Fig. 1. A delay element in the feedback loop of a continuous-time system satisfies the Internal Model Principle for periodic inputs. An infinite number of oscillators is required to replicate an arbitrary periodic input.

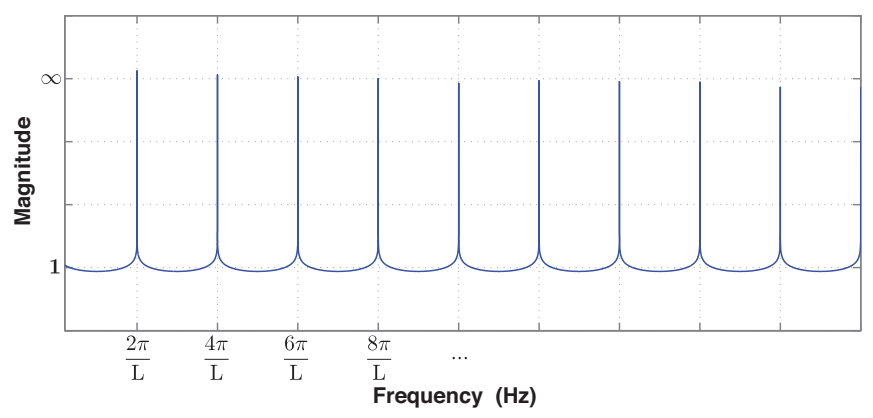

Fig. 2. Magnitude versus frequency for signal generator $\frac{\mathrm{e}^{-\mathrm{Ls}}}{1-\mathrm{e}^{-\mathrm{Ls}}}$.

trade-off is made between the robustness and high-frequency tracking performance when such filters are used. In order to improve the steady-state tracking performance, a phase compensator can be included to account for the phase of the filter as shown in references [18], [19]. The phase lead compensator can be easily implemented in discrete time.

In light of previous work on $\mathrm{RC}$, this work focuses on improving the design of an $\mathrm{RC}$ system that is robust and achieves minimum steady-state error. By incorporating a non-causal FIR filter in the RC loop, the robustness and high-frequency tracking performance of the system can be greatly improved compared to the current state-of-the art. Lastly, the performance of the proposed RC is demonstrated on a piezoelectric positioner used in an AFM system.

\section{Discrete Time Repetitive Control Design}

The discrete-time closed-loop system with $\mathrm{RC}$ is shown in Fig. 3. In the block diagram, the plant is represented by $G(z)$; $I(z)$ is the feedback controller, such as a PID controller; $Q(z)$ is a low-pass filter with a DC gain equal to 1 for robustness. This is required to filter out the infinite loop gains that are introduced by the memory loop at high-frequency harmonics. It also defines the control bandwidth of the $\mathrm{RC}$ and prevents the excitation of undesired dynamics at high frequencies. The filter $Q(z)$ can either be a Infinite Impulse Response (IIR) or Finite Impulse Response (FIR) filter. Alternately, a zero- 


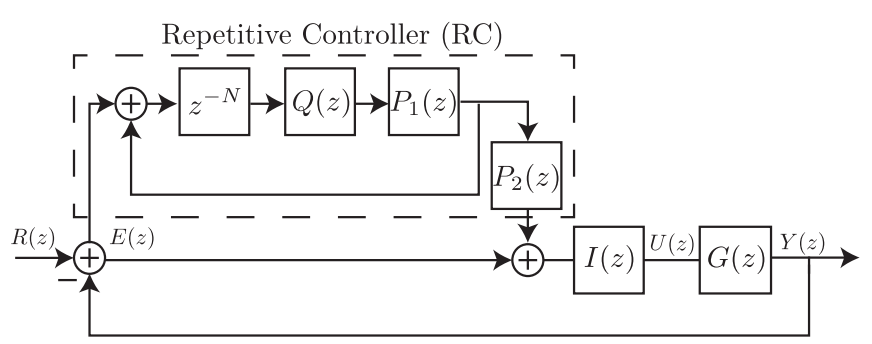

(a) The block diagram of the standard RC system.

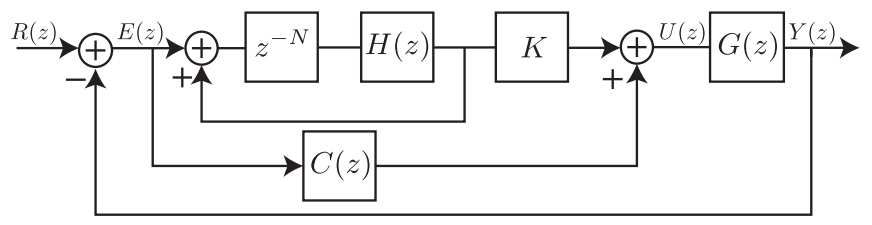

(b) The block diagram of the proposed RC system.

Fig. 3. Discrete-Time Repetitive Control System.

phase filter can be implemented in $Q(z)$. In [20], a zero phase error tracking control (ZPETC) is shown to perfectly track a reference with no phase delay, however, the method is based on pole-zero cancellation and phase cancellation, hence, the tracking performance is sensitive to modelling errors and plant parameter variations.

The performance of the RC is enhanced by including a positive phase lead compensator $P_{1}(z)$ and $P_{2}(z)$. The phase lead compensators are

$$
P_{1}(z)=z^{m 1}, P_{2}(z)=z^{m 2},
$$

where $m 1$ and $m 2$ are non-negative integers. The phase lead compensator $z^{m 1}$ and $z^{m 2}$ provides a linear phase lead.

$$
\theta_{1,2}(\omega)=m_{1,2} T_{s} \omega, \quad \omega \in\left(0, \pi / T_{s}\right) .
$$

In order to create an internal signal generator with period $T_{p}$, the repetitive controller in the inner loop contains a pure delay $z^{-N}$ where $N=T_{p} / T_{s}$ is the number of points per period $T_{p}$ and $T_{s}$ is the sampling time.

The stability of the closed-loop RC system shown in Fig. 3 was analysed in [21]. The transfer function relating the tracking error $E(z)$ and the reference signal $R(z)$ is

$$
\begin{aligned}
\frac{E(z)}{R(z)} & =\frac{1-W(z)}{1-W(z)+\left[\left(P_{2}(z)-1\right) W(z)+1\right] I(z) G(z)}, \\
& =\frac{[1-W(z)] S(z)}{1-W(z)\left[1-P_{2}(z) I(z) G(z) S(z)\right]},
\end{aligned}
$$

where $W(z)=Q(z) z^{\left(-N+m_{1}\right)}$ and $S(z)=1 /[1+I(z) G(z)]$. The first stability condition requires that the closed-loop system without RC $S(z)$, has no poles outside the unit circle in the $z$ plane. The second stability condition requires that $[1-W(z)]$ is stable. The third stability condition can be analysed using Small Gain Theorem [22] when,

$$
\left|W(z)\left[1-P_{2}(z) I(z) G(z) S(z)\right]\right|<1 .
$$

By satisfying condition (4) the closed-loop RC system is asymptotically stable.

\section{Proposed Discrete Time Repetitive Control DESIGN AND ANALYSIS}

The discrete-time closed-loop system with the proposed $\mathrm{RC}$ design is shown in Fig. 3(b). In the block diagram, the open-loop system is represented by $G(z) ; K$ is the RC gain; $C(z)$ is the feedback controller, such as a PID controller. $H(z)$ is a low-pass filter with a DC gain equal to 1 . In situations where the system's loop gain exceeds $180^{\circ}$, for example the first resonance frequency of the system, the RC controller must be attenuated to maintain stability. One option is to use an IIR filter as mentioned in Section II, however, IIR filters have a slow roll-off rate above the cut-off frequency and also introduce unwanted phase shift.

The ideal filter is one that provides an arbitrary response with unity gain and zero phase shift in the pass band and high attenuation in the stop-band. With such a filter, it would be possible to selectively include or exclude poles from the RC controller. This work describes a method for achieving an ideal filter response by employing a non-causal FIR filter. The filter is restricted to have symmetric coefficients and a length of precisely $2 N$ taps. In doing so, the time delay of the RC loop can be combined with the non-causal filter to create a causal filter with a pure time delay of $N$ samples. In other words, the FIR filter can implement both the RC time delay and an arbitrary frequency response which can be used to selectively include or exclude poles from the repetitive controller with zero phase shift.

A non-causal FIR filter can be described by

$$
y(k)=\sum_{i=-N}^{N} u(k-i) h(i),
$$

where $y(k)$ is the output, $u(k)$ is the input, and $h(i)$ is a vector of coefficients that are symmetric about $i=0$. The transfer function of (5) can be written,

$$
H(z)=\sum_{i=-N}^{N} z^{-i} h(i)
$$

The amplitude response of $H(z)$ can be arbitrary and the phase shift is zero due to the condition of symmetry. However, is it non-causal with a time-advance of $N$ samples. In Fig. 3(b), $H(z)$ is cascaded with the RC time delay $z^{-N}$, therefore, these two systems can be combined to create a causal FIR filter of length $2 N$ with a pure time delay of $N$ samples. That is,

$$
H(z)=\sum_{i=0}^{2 N} z^{-i} h(i-N)
$$

Therefore, the transfer function of the RC controller is simply

$$
G_{R C}(z)=K \frac{z^{-N} z^{N} H(z)}{1-z^{-N} z^{N} H(z)}=K \frac{H(z)}{1-H(z)},
$$

where $H(z)$ is the causal filter and $K$ is a constant gain.

Fig. 4(a) compares the response of an IIR and FIR loop filter with a cut-off frequency of $400 \mathrm{~Hz}$. Compared to the FIR filter, the IIR filter has a relatively slow roll-off and introduces a non-linear phase delay. The effect of both filters 


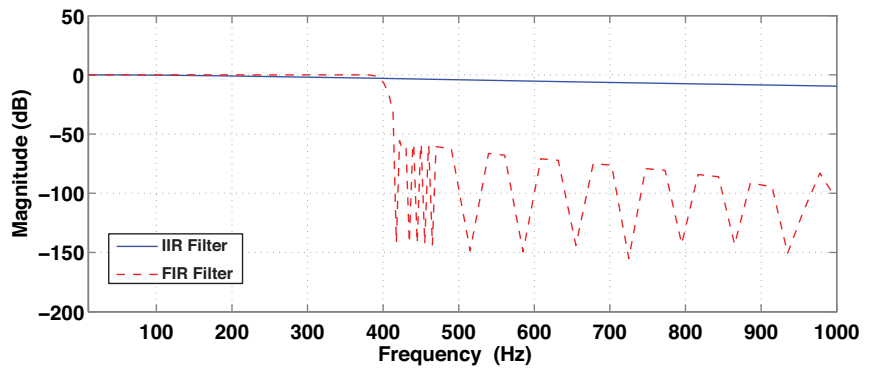

(a) Frequency response of a $1^{\text {st }}$ order low-pass IIR filter and the proposed FIR filter. Both the filters have a cut-off frequency of $400 \mathrm{~Hz}$.

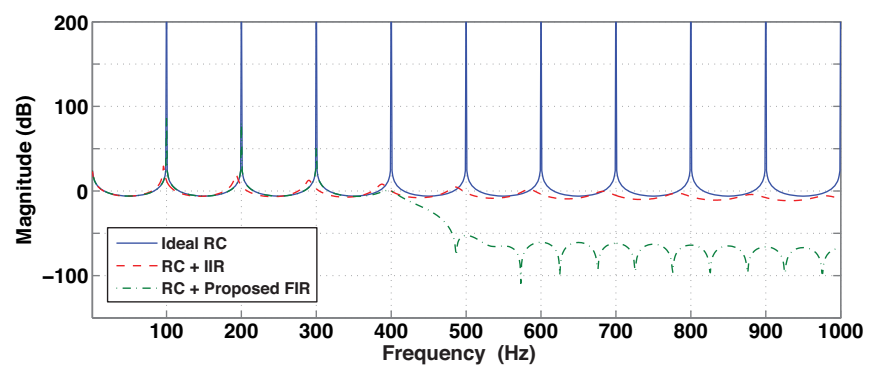

(b) Frequency respone of an ideal RC controller, RC controller with IIR filter and $\mathrm{RC}$ controller with the proposed FIR filter. The input periodic reference is $100 \mathrm{~Hz}$.

Fig. 4. The effects of stability filters on RC controllers.

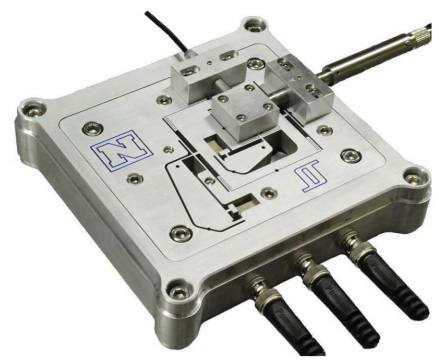

Fig. 5. Two-axis serial-kinematic nanopositioning platform with a range of $30 \mu \mathrm{m}$ designed and constructed at the EasyLab, University of Nevada, Reno, USA.

on the RC controller is illustrated in Fig. 4(b). The ideal $\mathrm{RC}$ controller has infinite gain at the harmonics as expected. However, if the system has a resonance frequency of $500 \mathrm{~Hz}$, the gain of the RC controller must be attenuated before this frequency. In the example, an IIR filter with a cutoff frequency of $400 \mathrm{~Hz}$ will cause instability. As a result, the cut-off frequency needs to be reduced which significant degrades the tracking performance of the system. On the other hand, with the proposed non-causal FIR filter, the unity gain and zero phase shift in the pass band does not degrade the ideal response of the $\mathrm{RC}$ controller.

\section{DESIGN OF RC FOR A NANOPOSITIONER}

In this section, the performance of three different controllers are compared for 1) PID control with notch filter 2) standard RC control with phase lead compensator and notch filter, and 3) proposed RC control. The details of the implementation and experimental results are presented

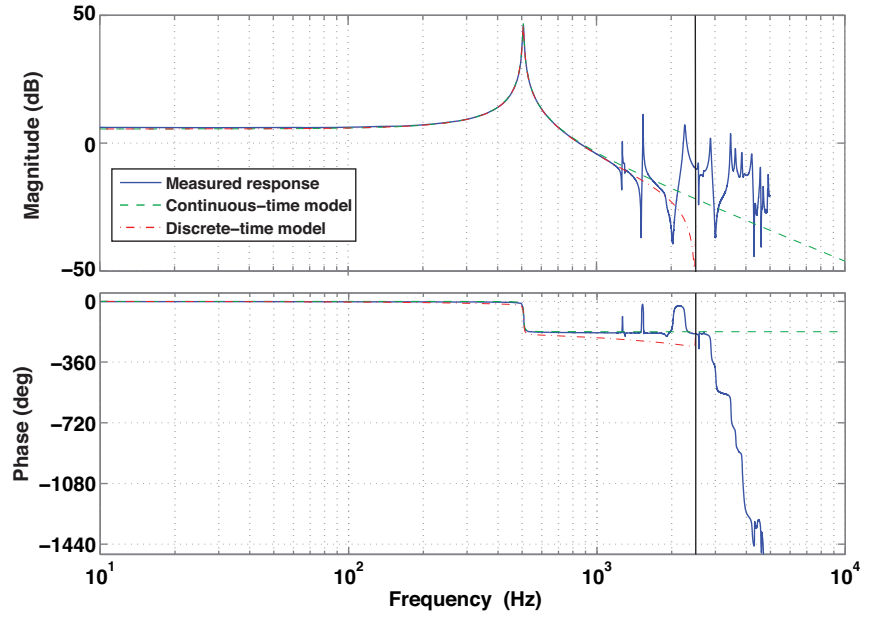

Fig. 6. The frequency response of the nanopositioner along the x-axis. The solid line (blue) is the measured response, the dash line (green) is the continuous-time model and the dash-dot line (red) is the linear discretetime model using Matlab function c2d with zero-order hold and sampling frequency of $5 \mathrm{kHz}$.

below. A model of the nanopositioner was first obtained, then a simulation study was conducted to study the effects of the $\mathrm{RC}$ parameters on the performance before implementing the discrete time $\mathrm{RC}$ on the nanopositioner.

\section{A. Nanopositioner Model}

The experiments were conducted on a two-axis serialkinematic nanopositioning stage pictured in Fig. 5. The nanopositioner was designed and constructed at the EasyLab, University of Nevada, Reno, USA. Each axis contains a $12 \mathrm{~mm}$ long piezoelectric stack actuator (Noliac NAC2003H12) with a free displacement of $12 \mu \mathrm{m}$ at $200 \mathrm{~V}$. The flexure design includes a mechanical amplifier to provide a total range of $30 \mu \mathrm{m}$. The flexures also mitigate crosscoupling so that each axis can be controlled independently. The position of the moving platform is measured by a Microsense 6810 capacitive sensor and 6504-01 probe, which has a sensitivity of $2.5 \mu \mathrm{m} / \mathrm{V}$. The stage is driven a PiezoDrive PDL200 voltage amplifier with a gain of 20 . The simulation and experiments are done along the $\mathrm{x}$-axis, which translates from left to right in Fig. 5 and has a resonance frequency of $509 \mathrm{~Hz}$.

For the purpose of control design, a second-order model is procured using the frequency domain least-squares technique. The model of the system is

$$
G(z)=\frac{0.09535 z+0.09526}{z^{2}-1.897 z+0.9972} .
$$

The frequency response of the model is compared to the experimental data in Fig. 6. The model closely approximates the first resonance mode which is sufficient for control design.

\section{B. PID Control with Notch Filter}

A popular technique for control of commercial nanopositioning systems is integral or proportional-integral control [23]. PID feedback controllers are commonly used to 
minimize hysteresis, creep, and the effect of vibration.

$$
P I D(s)=K_{p}+\frac{K_{i}}{s}+K_{d} s .
$$

The derivative term is rarely used due to the increased noise sensitivity and stability problems associated with high frequency resonance modes. On the contrary, PI and I controllers are simple to tune, however, the bandwidths of PI and I tracking controllers are severely limited by the presence of highly resonant modes.

Plant inversion techniques are popular as they are simple to implement and can provide a high closed-loop bandwidth if they are accurately tuned and the resonance frequency does not vary [24]. The transfer function of a typical inverse controller is

$$
N(s)=\frac{s^{2}+2 \zeta_{z} s+\omega_{z}^{2}}{\left(s+\omega_{z}\right)^{2}},
$$

where $\zeta_{z}$ and $\omega_{z}$ are the damping ratio and first resonance frequency of the nanopositioner. One of the crucial consideration with inversion based control is the effects of modelling error. For instance, if the resonance frequency falls below the frequency of the notch filter, the phase lag will cause the system to become unstable [24]. The notch filter is tuned to $509 \mathrm{~Hz}$ with an estimated damping $\zeta_{z}$ of 0.0045 .

The block diagram of the control system is similar to Fig. 3 with $P_{2}(z)=0$, in other words, the RC loop is absent. The controller $I(z)$ can be written

$$
I(z)=P I D(z) N(z) .
$$

The PID controller was tuned to provide acceptable gain and phase margins. The parameters of the controller are $K_{p}=0$, $K_{i}=500$ and $K_{d}=0$.

$$
P I D(z)=\frac{0.01 z+0.07}{z-1},
$$

In discrete time, the notch filter $N(z)$ was designed to invert the resonance frequency at $509 \mathrm{~Hz}$,

$$
N(z)=\frac{z^{2}-1.915 z+0.99}{z^{2}-1.454 z+0.5283} .
$$

The open-loop and closed-loop frequency response using PID control and notch filter are plotted in Fig. 7.

\section{Standard RC Control with Phase Lead Compensation and Notch Filter}

The block diagram of this control system is shown in Fig. 3. The first step is to design $I(z)$, the feedback controller without RC control. The controller can be written as

$$
I(z)=D(z) N(z)
$$

where $D(z)$ is a simple integral control and $N(z)$ is a notch filter similar to that in Section IV-B. The controller was tuned to provide adequate gain and phase margins. The implemented controller was

$$
D(z)=\frac{0.0025 z+0.0175}{z-1},
$$

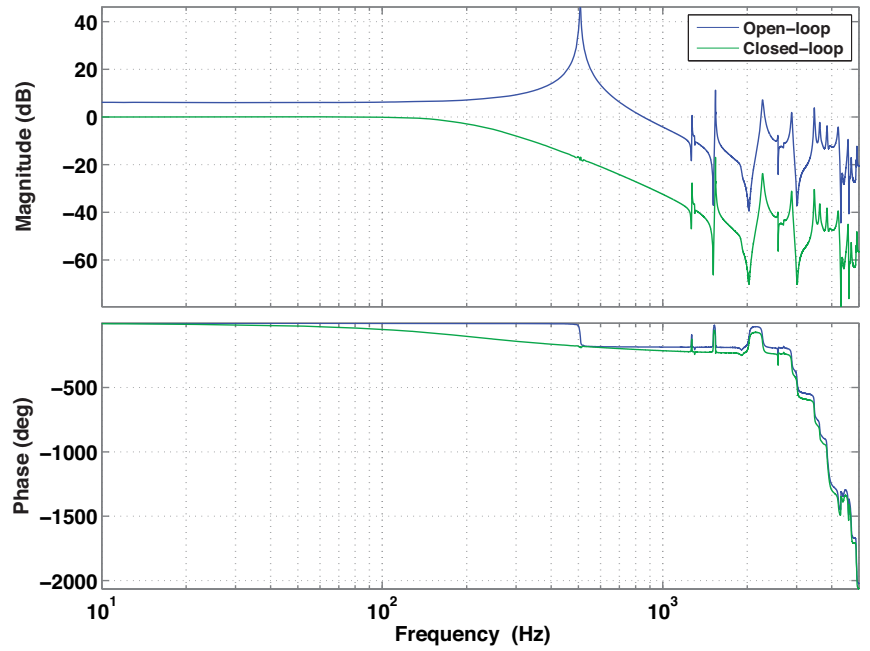

Fig. 7. Open-loop and closed-loop frequency response using PID control and notch filter.

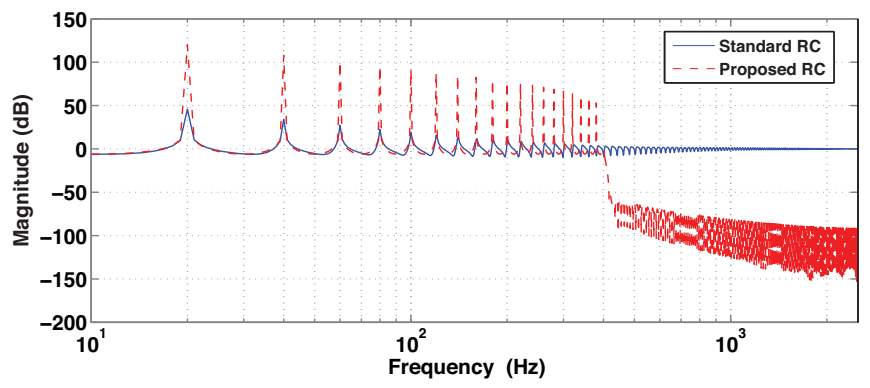

Fig. 8. Frequency response of the standard RC controller and the proposed $\mathrm{RC}$ controller.

and the notch filter $N(z)$ is identical to (14).

The next step is to design the RC controller. The signal period is $T_{p}=0.1 \mathrm{~s}$. The pure delay of the controller is set to $N=T_{p} / T_{s}=500$. The next step is to design a low-pass filter $Q(z)$. Here, a first-order Butterworth filter with a cut-off frequency of $200 \mathrm{~Hz}$ is chosen,

$$
Q(z)=\frac{0.1122+0.1122 z^{-1}}{1-0.7757 z^{-1}} .
$$

This filter allows for the reduction of gain at those frequency at which uncertainty exists and above the first resonance frequency. The phase lead compensators were chosen to be

$$
P_{1}(z)=z^{4}, P_{2}(z)=z^{4} .
$$

The frequency response of the standard RC controller shown here is given in Fig. 8. The open-loop and closed-loop frequency response using the standard RC control with phase lead compensation and notch filter is plotted in Fig. 9.

\section{Proposed RC Control}

A $2 \mathrm{~N}$-tap non-causal low-pass FIR filter with $N=500$ is designed. The cut-off frequency of is $400 \mathrm{~Hz}$. The feedback controller $C(z)$ is an integrator given as

$$
C(z)=\frac{0.0001}{z-1} \text {. }
$$



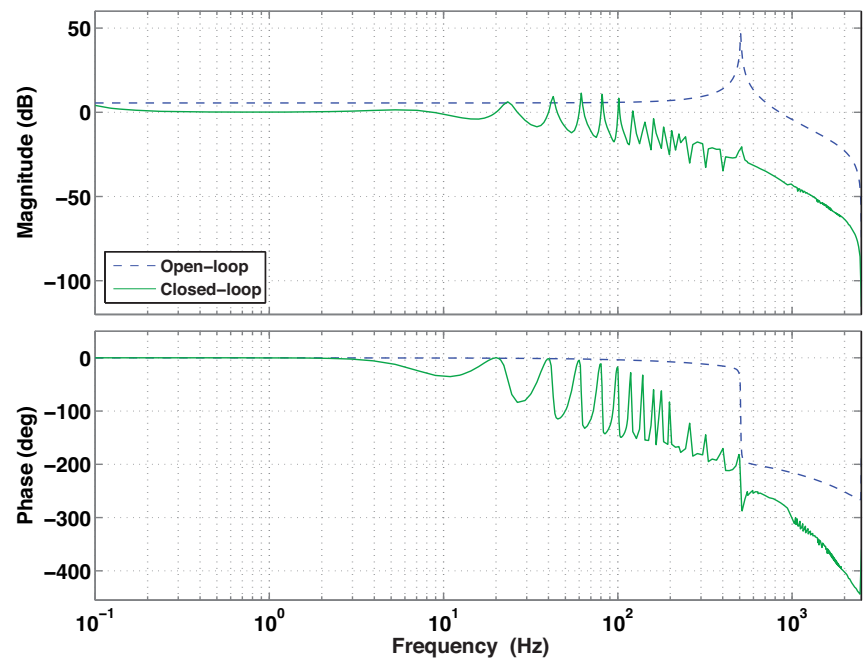

Fig. 9. Open-loop and closed-loop frequency response using standard RC control with phase lead compensation and notch filter.

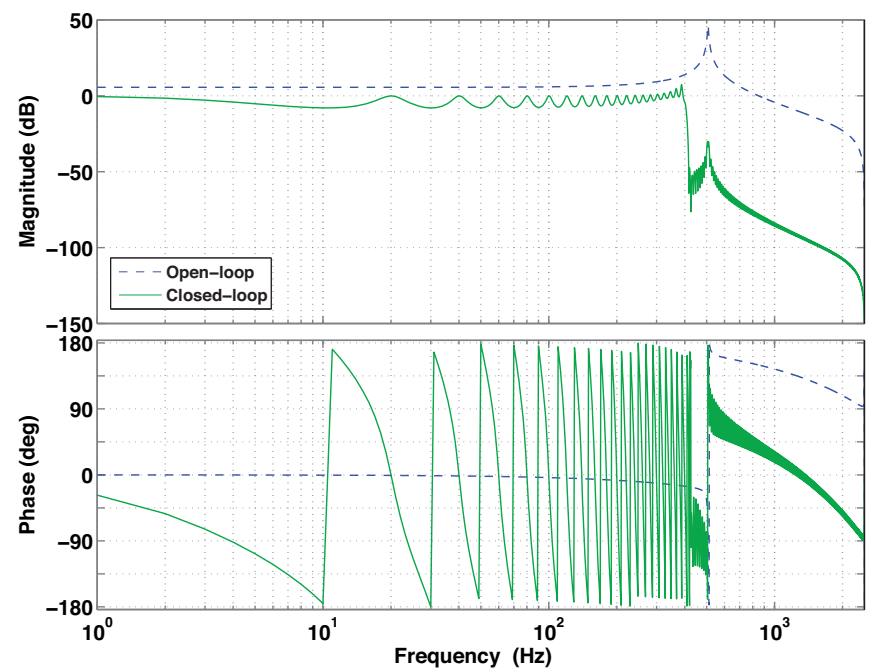

Fig. 10. Open-loop and closed-loop frequency response using proposed RC control.

The RC gain $K=0.3$. The frequency response of the proposed RC controller shown here is plotted in Fig. 8. The open-loop and closed-loop frequency response using the proposed RC control is plotted in Fig. 10.

\section{E. Experimental Results and Discussion}

In the experiment, the first reference signal was a $\pm 1 \mu \mathrm{m}$ sinusoidal wave at $20 \mathrm{~Hz}$. The tracking results for three control schemes are presented in Fig. 11 and Fig. 12(a). The second reference signal was a $\pm 1 \mu \mathrm{m}$ triangular wave at $20 \mathrm{~Hz}$. The tracking results are presented in Fig. 13 and Fig. 12(b). From the time signal in Fig. 11 and Fig. 13, the phase shift in the first controller is significant compared to the two RC control methods. From the error signal plot in Fig. 12(a) and Fig. 12(b), the performance of the proposed $\mathrm{RC}$ control is significantly better than the PID control with notch filter. The proposed RC control also outperforms the
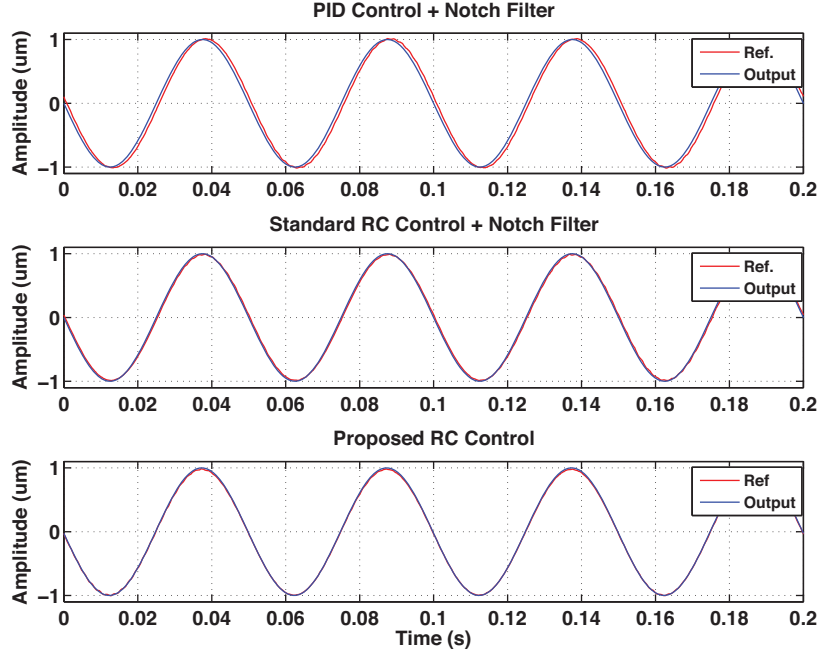

Fig. 11. The output displacement in response to a sinusoidal reference of $\pm 1 \mu m$ at $20 \mathrm{~Hz}$

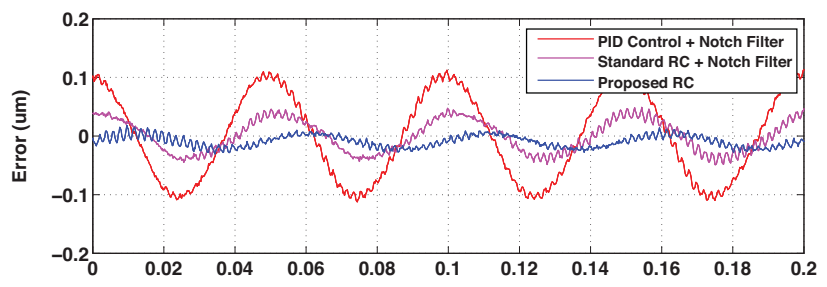

(a) Sinusoidal reference of $\pm 1 \mu \mathrm{m}$ at $20 \mathrm{~Hz}$.

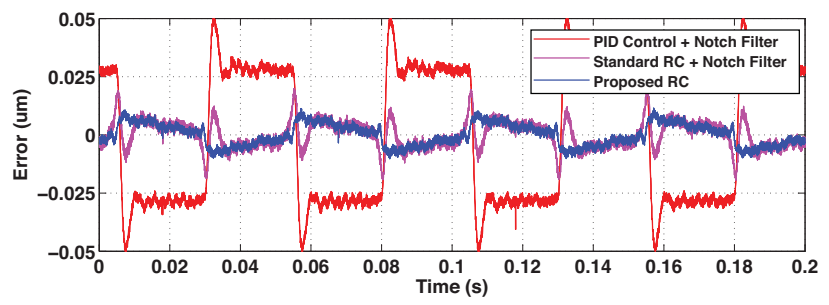

(b) Triangular reference of $\pm 1 \mu \mathrm{m}$ at $20 \mathrm{~Hz}$.

Fig. 12. Error in the output displacement.

standard RC control with phase lead compensation and notch filter.

An important factor that effects the RC performance is the amount of frequency shift due to the stability filters $Q(z)$, This is done by examining the denominator of the RC controllers. The transfer function of the standard RC control with phase lead compensator and notch filter is

$$
\begin{aligned}
R C_{1}(z) & =1+P_{2}(s) \frac{z^{-N} Q(z) P_{1}(z)}{1+z^{-N} Q(z) P_{1}(z)}, \\
& =\frac{1+z^{-N} Q(z) P_{1}(z)+P_{2}(s) z^{-N} Q(z) P_{1}(z)}{1+z^{-N} Q(z) P_{1}(z)},
\end{aligned}
$$

and the transfer function of the proposed $\mathrm{RC}$ control is

$$
R C_{2}(z)=\frac{z^{-N} H(z)}{1+z^{-N} H(z)} .
$$

The denominator of an ideal $\mathrm{RC}$ control is $\left(1+z^{-N}\right)$. The denominator of (20) indicates that the pole location is 

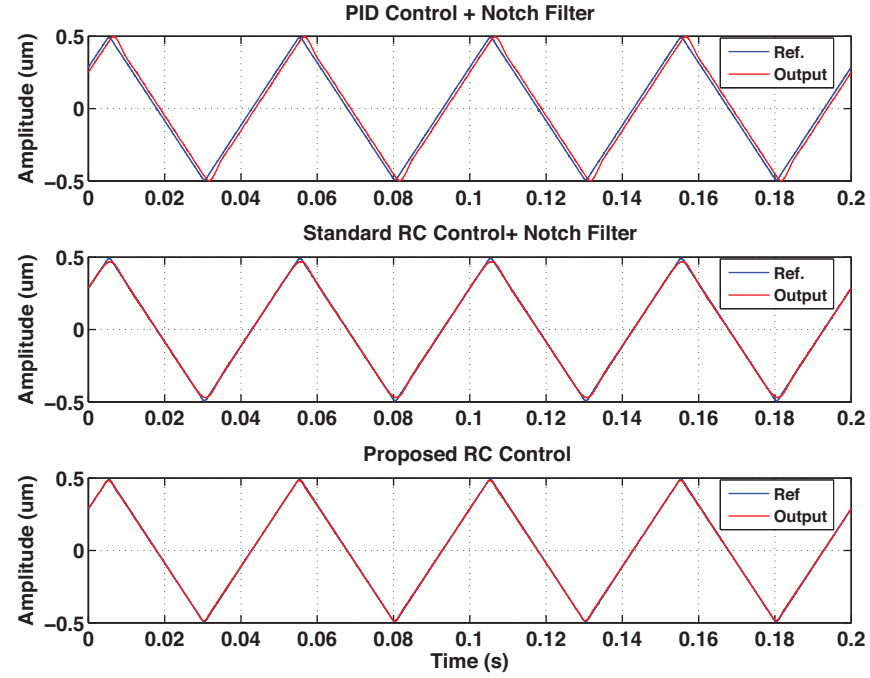

Fig. 13. The output displacement in response to a triangular reference of $\pm 1 \mu m$ at $20 \mathrm{~Hz}$.

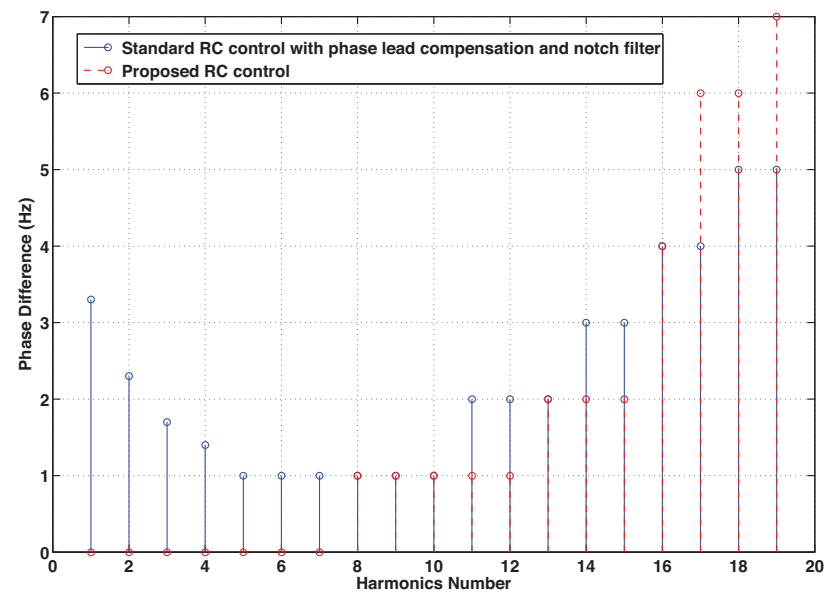

Fig. 14. Changes in pole location.

affected by the phase shift in filter $Q(z)$ and the phase lead compensator $P_{1}(z)$. The filter $H(z)$ is a $2 \mathrm{~N}$-tap non-causal low pass FIR filter which has zero phase shift. As a result, by observing the denominator of (21), the pole location of the system is not affected by the filter as shown in Fig. 14.

\section{CONCLUSIONS}

In this work, an RC control is incorporated with a novel filter design that greatly improves the high-frequency tracking performance without the requirement for phase compensators or plant inversion. The experiments were conducted on a piezoelectric positioner. The performance of the proposed RC design outperforms a PID controller with notch filter and standard RC control with phase lead compensation and notch filter. Future work includes plant inversion to further improve the performance.

\section{REFERENCES}

[1] Y. Wang, F. Gao, and F. J. Doyle III, "Survey on iterative learning control, repetitive control, and run-to-run control," Journal of Process Control, vol. 19, no. 10, pp. 1589-1600, 2009.
[2] M. Yamada, Z. Riadh, and Y. Funahashi, "Design of discrete-time repetitive control system for pole placement and application," Mechatronics, IEEE/ASME Transactions on, vol. 4, no. 2, pp. 110-118, 1999.

[3] C. H. Lee, "Robust repetitive control and application to a cd player," A disertation submitted for the degree of Doctor Philosophy, Trinity Hall Cambrigde, February 1998, 1998.

[4] D. H. Kim and T.-C. Tsao, "Robust performance control of electrohydraulic actuators for electronic cam motion generation," Control Systems Technology, IEEE Transactions on, vol. 8, no. 2, pp. 220227, 2000.

[5] Y. Shan and K. K. Leang, "Accounting for hysteresis in repetitive control design: Nanopositioning example," Automatica, vol. 48, pp. 1751-1758, 2012.

[6] A. J. Fleming and K. K. Leang, Design, Modeling and Control of Nanopositioning Systems. London, UK: Springer, 2014.

[7] J.-H. Moon, M.-N. Lee, and M. J. Chung, "Repetitive control for the track-following servo system of an optical disk drive," Control Systems Technology, IEEE Transactions on, vol. 6, no. 5, pp. 663-670, 1998.

[8] T. Inoue, M. Nakano, T. Kubo, S. Matsumoto, and H. Baba, "High accuracy control of a proton synchrotron magnet power supply," in Proceedings of the 8th World Congress of IFAC, vol. 20, 1981, pp. 216-221.

[9] T. Inoue, M. Nakano, and S. Iwai, "High accuracy control of servomechanism for repeated contouring," in 10th Annual Symp. on Incremental Motion Control Systems and Devices, 1981, pp. 285-292.

[10] B. A. Francis and W. M. Wonham, "The internal model principle of control theory," Automatica, vol. 12, no. 5, pp. 457-465, 1976.

[11] T.-C. Tsao and K.-K. Chew, "Analysis and synthesis of discrete-time repetitive controllers," Journal of Dynamic Systems, Measurement, and Control, vol. 111, p. 353, 1989.

[12] S. Hara, Y. Yamamoto, T. Omata, and M. Nakano, "Repetitive control system: a new type servo system for periodic exogenous signals," Automatic Control, IEEE Transactions on, vol. 33, no. 7, pp. 659668, 1988.

[13] G. Weiss, "Repetitive control systems: old and new ideas," Progress in Systems and Control Theory, vol. 22, pp. 389-404, 1997.

[14] L. Güvenç, "Stability and performance robustness analysis of repetitive control systems using structured singular values," Journal of dynamic systems, measurement, and control, vol. 118, no. 3, pp. 593-597, 1996.

[15] J. D. Ratcliffe, P. Lewin, E. Rogers, J. Hätönen, T. J. Harte, and D. H. Owens, "Stable repetitive control by frequency aliasing." in ICINCO, J. Filipe, J. Andrade-Cetto, and J.-L. Ferrier, Eds. INSTICC Press, 2005, pp. 323-327.

[16] R. Costa-Castello, J. Nebot, and R. Grinó, "Demonstration of the internal model principle by digital repetitive control of an educational laboratory plant," Education, IEEE Transactions on, vol. 48, no. 1, pp. 73-80, 2005.

[17] T. Inoue, "Practical repetitive control system design," in Decision and Control, 1990., Proceedings of the 29th IEEE Conference on. IEEE, 1990, pp. 1673-1678.

[18] H. L. Broberg and R. G. Molyet, "A new approach to phase cancellation in repetitive control," in Industry Applications Society Annual Meeting, 1994., Conference Record of the 1994 IEEE. IEEE, 1994, pp. 1766-1770.

[19] Y. Wang, D. Wang, B. Zhang, K. Zhou, and Y. Ye, "Robust repetitive control with linear phase lead," in American Control Conference, 2006. IEEE, 2006, pp. 6-pp.

[20] M. Tomizuka, "Zero phase error tracking algorithm for digital control," Trans. of ASME, Journal of Dynamic Systems, Measurement, and Control, vol. 109, pp. 65-68, 1987.

[21] U. Aridogan, Y. Shan, and K. K. Leang, "Design and analysis of discrete-time repetitive control for scanning probe microscopes," Journal of Dynamic Systems, Measurement, and Control, vol. 131, no. 6, p. 061103, 2009. [Online]. Available: http://link.aip.org/link/?JDS/131/061103/1

[22] K. Zhou and J. C. Doyle, Essentials of robust control. Prentice Hall Upper Saddle River, NJ, 1998, vol. 104.

[23] Y. Li, K. H. Ang, and G. Chong, "PID control system analysis and design," Control Systems, IEEE, vol. 26, no. 1, pp. 32-41, February 2006.

[24] K. K. Leang and S. Devasia, "Feedback-linearized inverse feedforward for creep, hysteresis, and vibration compensation in AFM piezoactuators," IEEE Transactions on Control Systems Technology, vol. 15, no. 5, pp. 927-935, September 2007. 